\section{Case Reports in Oncology}

\title{
Short-Term Outcome and MRI Changes in Three Adult Patients with Sickle Cell Disease and Aseptic Osteonecrosis after Treatment with Hyperbaric Oxygen Therapy: A Preliminary Report
}

\author{
Amr Shier $^{\mathrm{a}} \quad$ Mohamed Abdelrazek $^{\mathrm{b}} \quad$ Ashraf Soliman $^{c, d}$ \\ Vincenzo De Sanctis ${ }^{e} \quad$ Ahmed Elsayed ${ }^{f} \quad$ Mohamed Abdulla ${ }^{9}$ \\ Shehab Mohamed ${ }^{g} \quad$ Khadra Yassin $^{g} \quad$ Ilham Bilal $^{\mathrm{h}}$ Mohamed Yassin ${ }^{g}$ \\ aDepartment of Medical Education, Hamad General Hospital Doha, Doha, Qatar; \\ ${ }^{b}$ Department of Radiology, Hamad General Hospital Doha, Doha, Qatar; 'Division of \\ Endocrinology, Department of Pediatrics, Hamad General Hospital Doha, Doha, Qatar; \\ dDivision of Endocrinology, Department of Pediatrics, Alexandria University Children's \\ Hospital, Alexandria, Egypt; ePediatric and Adolescent Outpatient Clinic, Quisisana \\ Hospital, Ferrara, Italy; fDepartment of Orthopedic Surgery, Hamad General Hospital \\ Doha, Doha, Qatar; 9 Hematology Section/Medical Oncology (NCCCR), Hamad General \\ Hospital Doha, Doha, Qatar; h'Department of Pediatrics, Hamad General Hospital Doha, \\ Doha, Qatar
}

\section{Keywords}

Sickle cell disease - Avascular necrosis of bone · Hyperbaric oxygen

\section{Abstract}

Background: Musculoskeletal manifestations are common in sickle cell disease (SCD). Vasoocclusive crisis can manifest acutely as joint and bone pain, osteomyelitis and/or arthritis. It can also lead to chronic bone aches, bone deformities, degenerative arthritis, pathological fractures, and osteoporosis. Hyperbaric oxygen (HBO) therapy is a mode of treatment in which 
the patient is exposed to very high arterial and tissue oxygen pressure, during multiple sessions. It has been used as primary or adjunctive therapy for a variety of medical disorders, including necrotizing infection and sickle cell crisis. Case Report: In this case series, 3 patients with SCD and avascular necrosis were treated with 15-40 sessions of $\mathrm{HBO}$ and were assessed 6-12 months by MRI after treatment. They showed different clinical outcomes and MRI changes. Conclusion: We concluded that HBO can result in some subjective improvement, especially in early stages. Further studies on severe cases are needed.

(C) 2020 The Author(s)

Published by S. Karger AG, Basel

\section{Introduction}

Bone and joint complications can occur, over time, in about 31\% of patients with SCD, both in the acute setting such as painful vaso-occlusive crises (VOC) and as a source of chronic, progressive disability such as avascular necrosis (AVN), resulting in significant morbidities [1]. The major manifestations are osteonecrosis (ON), osteomyelitis, septic arthritis, and bone infarction [1].

Effective management of these bone and joint sequelae reflect accurate diagnosis, understanding of this disorder's pathophysiology, as well as knowledge of available medical and surgical treatment alternatives.

AVN can present with a broad spectrum of clinical manifestations ranging from incidental finding in asymptomatic patients to severe pain, functional limitations, and compromising the quality of life [1-4]. As $\mathrm{ON}$ is a progressive disease, its diagnosis may need a different form of radiological imaging, which ranges from standard X-ray, magnetic resonance imaging (MRI), and computed tomography (CT) to radionuclide scans. MRI is considered one of the best available imaging modalities to detect and to grade early bone changes. It might show areas of sclerosis and lucencies in the affected bone, articular surface collapse or flatting, attenuation of the epiphysis, and narrowing of joint space [5].

Early stages of the disease might benefit from nonoperative treatments. Hyperbaric oxygen (HBO) therapy has been suggested to reverse cellular ischemia and to decrease the inflammatory response for the symptomatic early stage ON. Furthermore, HBO helps facilitate the oxygenation of hypoxic bony tissue and to reduce the edema by increasing the concentration of dissolved oxygen [6]. We report the response of 3 patients with SCD and AVN to HBO therapy and reviewed the current literature.

\section{Case Presentation}

\section{Case 1}

A 33-year-old male patient had nontransfusion-dependent sickle cell disease (SCD) and was on hydroxyurea (1,000 mg p.o. daily) due to recurrent VOC complicated by AVN of both hips (pain score 7), his ferritin level was within normal limits. He received 28 daily sessions of HBO therapy (daily sessions which last $2 \mathrm{~h}$ with a pressure of $2.5 \mathrm{PSI}$ ). He reported marked improvement of hip pain (pain score 4), with concomitant improvement of MRI findings at 6 months after treatment (Fig. 1). 


\section{Case 2}

A 37-year-old female with nontransfusion-dependent SCD had infrequent VOC. She suffered from Graves' disease with ophthalmopathy and was on treatment with carbimazole and steroid. Her ferritin level was within normal limits. She developed AVN of the left shoulder (pain score 7) and received 15 sessions of HBO (daily sessions which last $2 \mathrm{~h}$ with pressure of 2.5 PSI). Subjectively, she felt minimal improvement in her shoulder pain (pain score 6), although her MRI done 1 year after her treatment showed significant partial improvement (Fig. 2).

\section{Case 3}

A 32-year-old female patient with transfusion-dependent SCD (received around 40 units of PRBCs over the last 5 years) was on the iron-chelating agent deferasirox and had a high level of ferritin $(1,680 \mu \mathrm{g} / \mathrm{L}$, normal level 12-160 $\mu \mathrm{g} / \mathrm{L})$. She had multiple VOC while on hydroxyurea therapy (500 mg twice daily) and developed AVN of both hips (pain score 6). She received 39 sessions of HBO (daily session, which last $2 \mathrm{~h}$ with pressure of $2.5 \mathrm{PSI}$ ). She reported no changes in her hip pain (pain score 6), and MRI of the hips done 1 year after treatment showed deterioration of the lesions (Fig. 3).

\section{Discussion}

Our study has concluded that HBO can result in some subjective improvement, especially in early stages, and can be helpful to treat early cases to relief their symptoms and prevent progression of AVN to advanced stage. However, further studies on severe cases are needed.

AVN is a specific form of $\mathrm{ON}$ driven by bone ischemia disrupting healthy bone remodeling $[2,3]$. The prevalence of ON shows a significant variation (up to $10 \%$ ) depending on age. Sickling of RBCs causes venous congestion, and thrombosis in the bone microvasculature resulting in ischemia that is enhanced by the increase of intraosseous pressure secondary to medullary hyperplasia $[4,5]$. Furthermore, elevated hemoglobin or hematocrit levels in combination with rheological problems lead to sickling and vaso-occlusion, especially in the femoral head sinusoids. The most frequent identified ON sites are the hip (74.6\%), shoulder, knee, and ankle $[4,5]$. The disease may involve the hips bilaterally, and it is common for $\mathrm{ON}$ to be asymptomatic, especially in early stages.

The AVN can present with a broad spectrum of clinical manifestations ranging from an incidental finding in asymptomatic patients to severe pain, functional limitations, and compromising of quality of life [1-4]. However, the imaging findings are similar in all forms, which pass through well-described radiological phases, regardless of the site of involvement.

ON can be diagnosed using different radiologic imaging modalities, ranging from standard radiograph, MRI, and CT to radionuclide scans. Radiolucency, sclerosis, or bone resorption are best seen on MRI and CT. On the other hand, degenerative changes related to collapse are easily detected on plain films. From the different radionuclear scan modalities, positron emission tomography (PET) provides a real-time image of the bony physiology. It is postulated that PET scans can detect ON even earlier than MRI and help to predict the disease progression [7].

Conservative treatment (such as pain control, bed rest with traction of the affected limb to relieve the weight, weight-bearing restriction, gradual weight-bearing activities, ambulatory assistive devices, and physiotherapy, including anti-gravity muscle strengthening exercises and a non-joint loading range of motion exercises) has been suggested only for early stages with tiny lesions or among patients where surgical treatment contraindicated [1-3]. 
Various medical therapies, including bisphosphonate, prostaglandin infusions, statins, and HBO therapy have also been proposed with variable results [8-14].

Nonoperative treatments might be very beneficial in the early stages of the disease, before ON progress to collapse of the femoral head articular surface.

When the deterioration of ON is rapid, operative intervention is necessary. Surgical intervention depends on the stage of the disease. In the early stages of ON, options include core decompression with or without bone grafting to attempt to prevent disease progression. In patients with severe pain and advanced disease, primary total hip arthroplasty (THA) is the most valid option to relieve the pain and restore functional impairment. Postoperative complications of THA include blood loss requiring transfusion, episodes of VOC, infections, and acute chest syndrome. Infection around the prosthesis is of particular concern for patients with SCD because they are more prone to recurrent bacteremia which may result in hematogenous seeding of the prosthesis [15-17].

In consideration of the current knowledge and the positive results of $\mathrm{HBO}$ for the treatment of sickle cell crisis [18], priapism [19], leg ulcers [20], and central retinal artery occlusion (CRAO) [21], we treated our 3 young adult patients with SCD and AVN with HBO. Two out of the 3 patients had positive results to treatment.

A study published by Camporesi et al. [6] reported a significant pain improvement, after 20 oxygen treatments for 6 weeks in a hyperbaric chamber, in 20 patients with unilateral femoral head necrosis. All patients remained symptoms free in the 7-year follow-up period and none of them required hip arthroplasty. Substantial radiographic healing of the $\mathrm{ON}$ was observed in 7 of 9 hips. Similar results were reported by Moon et al. [22]. The authors selected 12 patients who suffered from early-stage AVN of the head of the femur (four bilateral); they received daily HBO therapy for 100 days. Overall, $81 \%$ of patients who received this therapy showed a normal MRI scan compared to $17 \%$ in the control group.

HBO therapy is a way to deliver very high arterial oxygen tension ranging between 1,000 and $1,600 \mathrm{~mm} \mathrm{Hg}$ to achieve tissue oxygen tension around $400 \mathrm{~mm} \mathrm{Hg}$ by exposing the patient to very high oxygen tension in a closed chamber over multiple sessions (ranging from 2 to 40 sessions) [22].

The use of HBO leads to increased arterial oxygen tension that may reverse RBCs sickling in the microcirculation. Also, the elevated level of pO2 may decrease the risk of tissue ischemia and infarction [23]. HBO can exert its beneficial effects on AVN by enhancing the molecular signaling that helps accelerate bone remodeling. It also promotes the osteogenic differentiation of mesenchymal stem cells [24]. HBO also inhibits osteoclast formation and bone resorption [25]. HBOT can have some anti-inflammatory effect in patients with AVN as it lowers the level of IL-1b, TNF-a, and IL-6, which are key cytokines for bone resorption.

These effects can explain the resolution of edema seen in radiological imaging and the relief of AVN symptoms by decreasing the bone internal pressure, restoring venous return, and improving microcirculation [26]. Furthermore, HBO can promote the viability and activity of the phagocytic cells which are beneficial to the absorption and removal of necrotic bone tissue [27].

Side effects of HBO therapy include problems in the middle ear pressure equalization, sinus, and tooth squeeze. These complications are correlated strongly to the number of treatment sessions received [28]. Ambiru et al. [28] reported a complication rate of $8.1 \%$ in patients that received 11-29 treatments, the percentage was higher (17.1\%) when the number of $\mathrm{HBO}$ treatments was $\geq 30$. However, none of these side effects occurred in our patients.

Strengths of our study include studying of AVN cases secondary to SCD which is considered as a very specific study and the first study done in the Middle Eastern population. 
Limitations of our study include the small number of patients in the study which decreases the power of our study. Our study was performed on middle-age patients which can make it difficult to generalize the results to include a population of children.

In summary, although it is not possible to draw any therapeutic conclusions for using HBO therapy in the management of $\mathrm{ON}$ in patients with SCD, further studies are encouraged to assess its safety and efficacy.

\section{Acknowledgement}

The authors acknowledge the Qatar National Library (QNL) for financial support and internal medicine residency program for scientific support.

\section{Statement of Ethics}

These case series were approved by the Hamad Medical Corporation Medical Research Centre and consents were obtained from all patients.

\section{Disclosure Statement}

The authors declare no potential conflicts of interest concerning the research, authorship, and/or publication of this article.

\section{Funding Sources}

Qatar National Library (QNL).

\section{Authors Contributions}

Amr A Shier, Mohammed Abdelrazek, Ashraf Soliman, Vincenzo De Sanctis and Mohamed Yassin: writing and editing. Ahmed Elsayed, Mohamed AJ Abdulla, Shehab Mohamed, Khadra Yassin, Ilham Bilal: Clinical care.

\section{References}

1 Matos MA, dos Santos Silva LL, Brito Fernandes R, Dias Malheiros C, Pinto da Silva BV. Avascular necrosis of the femoral head in sickle cell disease patients. Ortop Traumatol Rehabil. 2012 Mar-Apr;14(2):155-60.

2 Milner PF, Kraus AP, Sebes JI, Sleeper LA, Dukes KA, Embury SH, et al. Sickle cell disease as a cause of osteonecrosis of the femoral head. N Engl J Med. 1991 Nov;325(21):1476-81.

3 Lafforgue P. Pathophysiology and natural history of avascular necrosis of bone. Joint Bone Spine. 2006 Oct;73(5):500-7.

4 Daltro G, Franco BA, Faleiro TB, Rosário DA, Daltro PB, Fortuna V. Osteonecrosis in sickle cell disease patients from Bahia, Brazil: a cross-sectional study. Int Orthop. 2018 Jul;42(7):1527-34.

5 Petek D, Hannouche D, Suva D. Osteonecrosis of the femoral head: pathophysiology and current concepts of treatment. EFORT Open Rev. 2019 Mar;4(3):85-97. 
6 Camporesi EM, Vezzani G, Bosco G, Mangar D, Bernasek TL. Hyperbaric oxygen therapy in femoral head necrosis. J Arthroplasty. 2010 Sep;25(6 Suppl):118-23.

7 Steinberg ME, Hayken GD, Steinberg DR. A quantitative system for staging avascular necrosis. J Bone Joint Surg Br. 1995 Jan;77(1):34-41.

8 Agarwala S, Banavali SD, Vijayvargiya M. Bisphosphonate Combination Therapy in the Management of Postchemotherapy Avascular Necrosis of the Femoral Head in Adolescents and Young Adults: A Retrospective Study From India. J Glob Oncol. 2018 Sep;4(4):1-11.

9 Disch AC, Matziolis G, Reinke P, Perka C. Intravenous Iloprost treatment for severe bone pain caused by sickle cell crisis. Thromb Haemost. 2004 May;91(5):1047-9.

10 Disch AC, Matziolis G, Perka C. The management of necrosis-associated and idiopathic bone-marrow oedema of the proximal femur by intravenous iloprost. J Bone Joint Surg Br. 2005 Apr;87(4):560-4.

11 Laroche M, Jacquemier JM, Montane de la Roque P, Arlet J, Mazières B. [Nifedipine per os relieves the pain caused by osteonecrosis of the femur head]. Rev Rhum Mal Osteoartic. 1990 Oct;57(9):669-70. French.

12 Pritchett JW. Statin therapy decreases the risk of osteonecrosis in patients receiving steroids. Clin Orthop Relat Res. 2001 May;386:173-8.

13 Bernbeck B, Christaras A, Krauth K, Lentrodt S, Strelow H, Schaper J, et al. Bone marrow oedema and aseptic osteonecrosis in children and adolescents with acute lymphoblastic leukaemia or non-Hodgkin-lymphoma treated with hyperbaric-oxygen-therapy (HBO): an approach to cure? - BME/AON and hyperbaric oxygen therapy as a treatment modality. Klin Padiatr. 2004 Nov-Dec;216(6):370-8.

14 Koren L, Ginesin E, Melamed Y, Norman D, Levin D, Peled E. Hyperbaric oxygen for stage I and II femoral head osteonecrosis. Orthopedics. 2015 Mar;38(3):e200-5.

15 Hernigou P, Bachir D, Galacteros F. The natural history of symptomatic osteonecrosis in adults with sicklecell disease. J Bone Joint Surg Am. 2003 Mar;85(3):500-4.

16 Kamath AF, McGraw MH, Israelite CL. Surgical management of osteonecrosis of the femoral head in patients with sickle cell disease. World J Orthop. 2015 Nov;6(10):776-82.

17 Johannson HR, Zywiel MG, Marker DR, Jones LC, McGrath MS, Mont MA. Osteonecrosis is not a predictor of poor outcomes in primary total hip arthroplasty: a systematic literature review. Int Orthop. 2011 Apr;35(4):465-73.

18 Stirnemann J, Letellier E, Aras N, Borne M, Brinquin L, Fain O. Hyperbaric oxygen therapy for vaso-occlusive crises in nine patients with sickle-cell disease. Diving Hyperb Med. 2012 Jun;42(2):82-4.

19 Azık FM, Atay A, Kürekçi AE, Ay H, Kibar Y, Ozcan O. Treatment of Priapism with Automated Red Cell Exchange and Hyperbaric Oxygen in an 11-year-old Patient with Sickle Cell Disease. Turk J Haematol. 2012 Sep;29(3):270-3.

20 Campton-Johnston S, Wilson J, Ramundo JM. Treatment of painful lower extremity ulcers in a patient with sickle cell disease. J Wound Ostomy Continence Nurs. 1999 Mar;26(2):98-104.

21 Canan H, Ulas B, Altan-Yaycioglu R. Hyperbaric oxygen therapy in combination with systemic treatment of sickle cell disease presenting as central retinal artery occlusion: a case report. J Med Case Reports. 2014 Nov;8(1):370.

22 Moon RE, Feldmeier JJ. Hyperbaric oxygen: an evidence based approach to its application. Undersea Hyperb Med. 2002;29(1):1-3.

23 Wallyn CR, Jampol LM, Goldberg MF, Zanetti CL. The use of hyperbaric oxygen therapy in the treatment of sickle cell hyphema. Invest Ophthalmol Vis Sci. 1985 Aug;26(8):1155-8.

24 Lin SS, Ueng SW, Niu CC, Yuan LJ, Yang CY, Chen WJ, et al. Effects of hyperbaric oxygen on the osteogenic differentiation of mesenchymal stem cells. BMC Musculoskelet Disord. 2014 Feb;15(1):56.

25 Al Hadi H, Smerdon GR, Fox SW. Hyperbaric oxygen therapy suppresses osteoclast formation and bone resorption. J Orthop Res. 2013 Nov;31(11):1839-44.

26 Bosco G, Vezzani G, Mrakic Sposta S, Rizzato A, Enten G, Abou-Samra A, et al. Hyperbaric oxygen therapy ameliorates osteonecrosis in patients by modulating inflammation and oxidative stress. J Enzyme Inhib Med Chem. 2018 Dec;33(1):1501-5.

27 Li W, Ye Z, Wang W, Wang K, Li L, Zhao D. Clinical effect of hyperbaric oxygen therapy in the treatment of femoral head necrosis: A systematic review and meta-analysis. Orthopade. 2017 May;46(5):440-6.

28 Ambiru S, Furuyama N, Aono M, Otsuka H, Suzuki T, Miyazaki M. Analysis of risk factors associated with complications of hyperbaric oxygen therapy. J Crit Care. 2008 Sep;23(3):295-300. 


\section{Case Reports in Oncology}
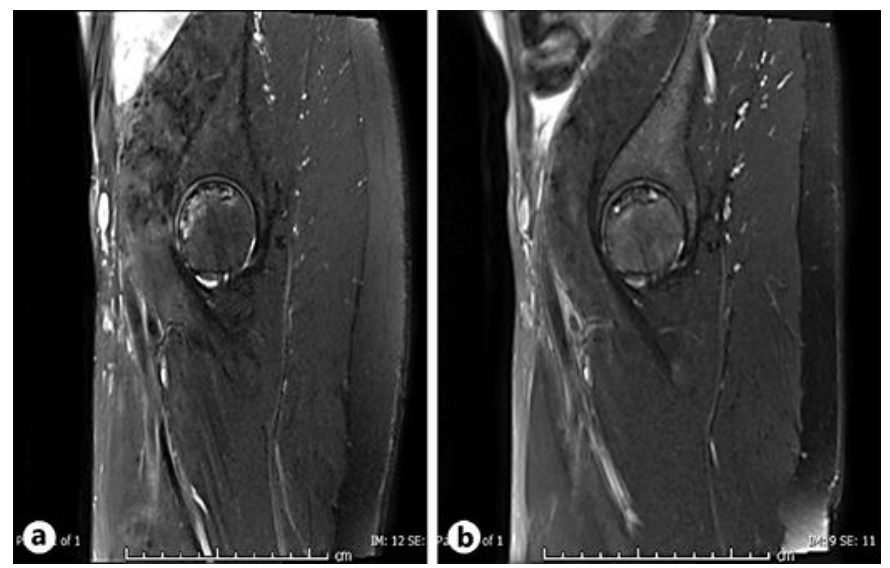

Fig. 1. a Sagittal STIR (short T1 inversion recovery) of the left hip joint before treatment with HBO shows geographical subarticular area of high-signal intensity in the head of femur involving $45 \%$ of the femoral head (stage IC, severe). No detected structural collapse of the head. b Sagittal STIR of the left hip after treatment shows that decrease in the subarticular is of high-signal intensity involving about $25 \%$ of the femoral head (stage IB, moderate) denoting improvement of the condition.
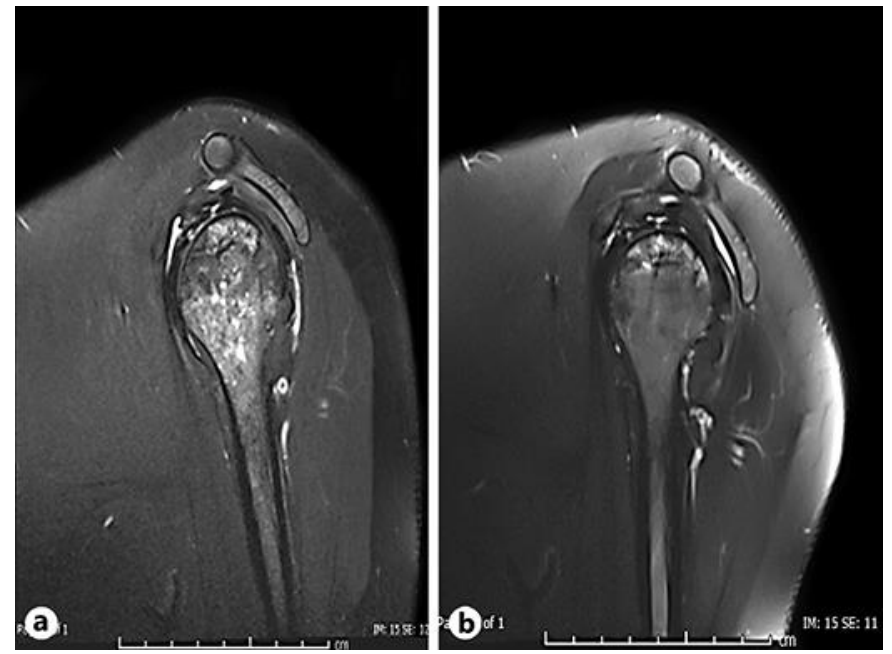

Fig. 2. a Sagittal T2 fat suppressed image of the left shoulder before treatment with hyperbaric oxygen shows subarticular geographical areas of high-signal intensity involving about $45 \%$ of the head of the humerus (stage IC, severe). No detected structural collapse of the head. Also noted geographical high-signal intensity in the proximal shaft. b Sagittal T2 fat suppressed image of the left shoulder after treatment shows nearly stable appearance of subarticular areas of high-signal intensity in the head of the humerus. However, significant improvement is noted in the proximal shaft with absence of high-signal intensity. 


\section{Case Reports in Oncology}
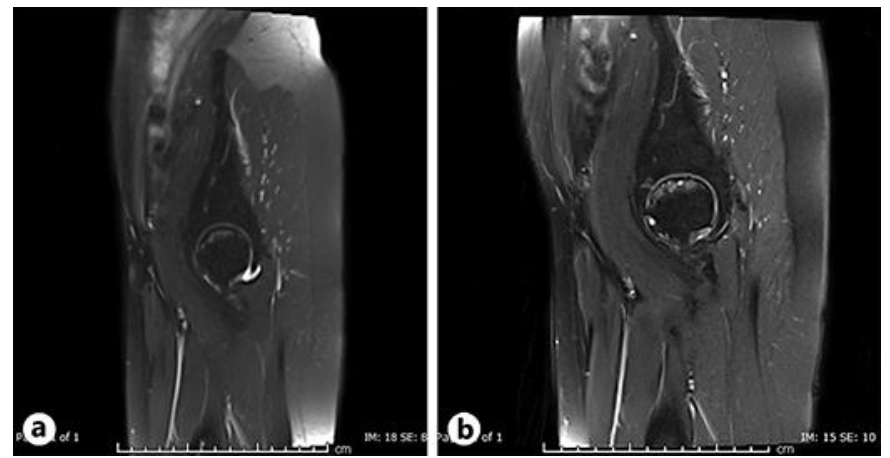

Fig. 3. a Sagittal STIR (short T1 inversion recovery) of the left hip joint before treatment with hyperbaric oxygen shows geographical subarticular areas of high-signal intensity in the head of the femur involving $35 \%$ of the femoral head with no definite collapse (stage IC, severe). b Sagittal STIR of the left hip after treatment shows an increase in the areas of high-signal intensity involving now about $45 \%$ of the femoral head with subchondral cystic changes (stage IIC, severe) denoting worsening of the condition. 